Bull. Austral. Math. Soc.

VOL. 41 (1990) [135-147]

\title{
SLANT IMMERSIONS
}

\section{BANG-YeN ChEN}

\begin{abstract}
A slant immersion is defined as an isometric immersion from a Riemannnian manifold into an almost Hernitian manifold with constant Wirtinger angle. In this article we give some fundamental results concerning slant immersions. Several results on slant surfaces in $C^{2}$ are also proved.
\end{abstract}

\section{INTRODUCTION}

Let $N$ be an $n$-dimensional Reimannian manifold and $M$ an almost Hermitian manifold with almost complex structure $J$ and with almost Hermitian metric $g$. An isometric immersion $f: N \rightarrow M$ is called holomorphic if, for any point $x$ in $N$, we have $J\left(T_{x} N\right)=T_{x} N$, where $T_{x} N$ denotes the tangent space of $N$ at $x$. The immersion $f$ is called totally real (see [3]) (or Lagrangian) if we have $J\left(T_{x} N\right) \subseteq T_{x}^{\perp} N$ for each $x \in N$, where $T_{x}^{\perp \perp} N$ denotes the normal space of $N$ in $M$ at $x$. In the following, we denote by $\langle$,$\rangle the inner product for N$ as well as for $M$.

For any vector $X$ tangent to $N$, we put

$$
J X=P X+F X
$$

where $P X$ and $F X$ denote the tangential and the normal components of $J X$, respectively. Then $P$ is an endomorphism of the tangent bundle $T N$ and $F$ a normal-bundlevalued 1-form on $T N$. For each non-zero vector $X$ tangent to $N$ at $x$ the angle $\theta(X)$ between $J X$ and $T_{x} N$ is called the Wirtinger angle of $X$. In the following we call an immersion $f: N \rightarrow M$ a general slant immersion if the Wirtinger angle $\theta(X)$ is a constant (which is independant of the choice of $x \in N$ and $X \in T_{x} N$ ). Holomorphic and totally real immersions are general slant immersions with Wirtinger angle $\theta$ equal to 0 and $\pi / 2$ respectively. A general slant immersion which is not holomorphic is simply called a slant immersion. In this case, the Wirtinger angle $\theta$ is called the slant angle of the slant immersion.

A slant submanifold is said to be proper if it is not totally real. Finally, a proper slant submanifold is said to be Kaehlerian slant if the endomorphism $P$ is parallel, that is, $\nabla P=0$. A Kaehlerian slant submanifold is a Kaehler manifold with respect to the

Received 1 March, 1989

Copyright Clearance Centre, Inc. Serial-fee code: 0004-9729/90 \$A2.00+0.00. 
induced metric and the almost complex structure $\stackrel{0}{J}=(\sec \theta) P$ where $\theta$ is the slant angle.

In this paper, we give some fundamental properties of slant immersions. Some relations between submanifolds with holomorphic submanifolds or totally real submanifolds are obtained. Furthermore, many results concerning slant surfaces in $\mathrm{C}^{2}$ are proved.

\section{KAEHLERIAN SLANT IMMERSION AND $\nabla P=0$}

Let $N$ be an $n$-dimensional Riemannian manifold isometrically immersed in an almost Hermitian manifold $M$. (All manifolds are assumed to be connected unless otherswise mentioned.) Let $P$ be the endomorphism defined by (1.1). Since $M$ is almost Hermitian, we have $\langle P X, Y\rangle+\langle X, P Y\rangle=0$. Thus $P^{2}$, which is simply denoted by $Q$, is self-adjoint. Therefore, each tangent space $T_{x} N$ of $N$ at $x$ admits the following orthogonal direct decomposition of eigenspaces of $Q$ :

$$
T_{x} N=\mathcal{D}_{x}^{1} \oplus \ldots \oplus \mathcal{D}_{x}^{k(x)}
$$

Since $P$ is skew-symmetric and $J^{2}=-1$, each eigenvalue $\lambda_{i}$ of $Q=P^{2}$ lies in $[-1,0]$ and moreover, if $\lambda_{i} \neq 0$, the corresponding eigenspace $\mathcal{D}_{x}^{i}$ is of even dimension and it is invariant under the endomorphism $P$, that is $P\left(\mathcal{D}_{x}^{i}\right)=\mathcal{D}_{x}^{i}$. Furthermore, for each $\lambda_{i} \neq-1, \operatorname{dim} F\left(\mathcal{D}_{x}^{i}\right)=\operatorname{dim} \mathcal{D}_{x}^{i}$ and the normal subspaces $F\left(D_{x}^{i}\right)$, for $i=$ $1, \ldots, k(x)$ are mutually perpendicular. Thus we have $\operatorname{dim} M \geq 2 \operatorname{dim} N-\operatorname{dim} \mathcal{H}_{x}$ where $\mathcal{H}_{x}$ denotes the eigenspace of $Q$ with eigenvalue -1 .

The following lemma follows from the definition of $\nabla Q$ which is defined by $\left(\nabla_{X} Q\right) Y=\nabla_{X}(Q Y)-Q\left(\nabla_{X} Y\right)$ for $X, Y$ in $T N$.

LEMm A 2.1. Let $N$ be a submanifold of an almost Hermitian manifold $M$. Then the self-adjoint endomorphism $Q\left(=P^{2}\right)$ is parallel if and only if

(1) each eigenvalue $\lambda_{i}$ of $Q$ is constant on $N$;

(2) each distribution $\mathcal{D}^{i}$ (associated with $\lambda_{i}$ ) is completely integrable and

(3) $N$ is locally the Riemannian product of the leaves of the distributions.

Since this lemma can be proved in a standard way and is somewhat well-known, we omit its proof.

By using Lemma 2.1 we have the following characterisation of submanifolds with $\nabla P=0$.

Lemma 2.2. Let $N$ be a submanifold of an almost Hermitian manifold $M$. Then $\nabla P=0$ if and only if $N$ is locally the Riemannian product $N_{1} \times \ldots \times N_{k}$, where each $N_{i}$ is either a Kaehler submanifold, a totally real submanifold, or a Kaehlerian slant submanifold. 
Proof: Under the hypothesis, if $P$ is parallel, then $Q$ is parallel. Thus Lemma 2.1 implies that $N$ is locally the Riemannian product $N_{1} \times \ldots \times N_{k}$ of the leaves of the distributions defined by eigenvalues of $Q$ and moreover, each eigenvalue $\lambda_{i}$ is constant on $N$. If an eigenvalue $\lambda_{i}$ is zero, $N_{i}$ is totally real. If $\lambda_{i}$ is -1 then $N_{i}$ is a holomorphic submanifold. If $\lambda_{i} \neq 0,-1$, then, because $\mathcal{D}^{i}$ is invariant under the endomorphism $P$ and $\langle P X, Y\rangle=-\lambda_{i}\langle X, Y\rangle$ for $X, Y$ in $\mathcal{D}^{i}$, we have $|P X|=$ $\sqrt{-\lambda_{i}}|X|$. Thus the Wirtinger angle $\theta(X)$ satisfies $\cos \theta(X)=\sqrt{-\lambda_{i}}$, which is a constant $\neq 0$, or -1 . Therefore, $N_{i}$ is a proper slant submanifold. Now assume $\lambda_{i} \neq 0$ and put $P_{i}=\left.P\right|_{T N_{i}}$. Then $P_{i}$ is simply the endomorphism of $T N_{i}$ induced from $J$. Let $\nabla^{i}$ denote the Riemannian connection on $N_{i}$. Since $N_{i}$ is totally geodesic in $N$, we obtain $\left(\nabla_{X}^{i} P_{i}\right) Y=\left(\nabla_{X} P\right) Y=0$ for $X, Y$ tangent to $N_{i}$. This shows that if $N_{i}$ is holomorphic, then $N_{i}$ is a Kaehler submanifold. And if $N_{i}$ is proper slant, $N_{i}$ is a Kaehlerian slant submanifold of $M$.

The converse can be verified directly.

From Lemma 2.2 we obtain the following.

Proposition 2.3. Let $N$ be an irreducible submanifold of an almost Hermitian manifold $M$. If $N$ is neither holomorphic nor totally real, then $N$ is a Kaehlerian slant submanifold if and only if $P$ is parallel, that is, $\nabla P=0$.

Proposition 2.4. Let $N$ be a surface in an almost Hermitian manifold $M$. If $N$ is neither totally real nor holomorphic in $M$, then the following three statements are equivalent:

(a) $\nabla P=0$, that is, $P$ is parallel;

(b) $N$ is a Kaellerian slant surface;

(c) $N$ is a proper slant surface.

ProOF: Since each proper slant submanifold is of even dimension, Lemma 2.2 implies that if $P$ is parallel, then $N$ is a Kaehler surface, or a totally real surface or a Kaehlerian slant surface. Since $N$ is assumed to be neither totally real nor holomorphic, we see that (a) and (b) are equivalent under the hypothesis. It is obvious that (b) implies (c). Now we prove (c) implies (b). Let $N$ be a proper slant surface in $M$ with slant angle $\theta$. We may choose an orthonormal frame $e_{1}, e_{2}$ tangent to $N$ such that $P e_{1}=(\cos \theta) e_{2}$ and $P e_{2}=-(\cos \theta) e_{1}$. Then we have $\left(\nabla_{X} P\right) e_{1}=\cos \theta\left(\omega_{2}^{1}(X)+\omega_{1}^{2}(X)\right) e_{1}$, where $\omega_{i}^{j}$ denotes the connection form on $N$ defined by $d e_{i}=\sum \omega_{i}^{j} \otimes e_{j}$. Since $\omega_{2}^{1}=-\omega_{1}^{2}$, we obtain $\nabla P=0$.

3. Minimality and slant immersions with $\nabla F=0$.

Let $N$ be a submanifold in a Kaehler manifold $M$. We denote by $\nabla$ and $\tilde{\nabla}$ the Riemannian connections of $N$ and $\widetilde{M}$ respectively. By $A, D$ and $\sigma$ the Weingarten 
map, the normal connection and the second fundamental form of $N$ in $M$, respectively. Then the Gauss and Weingarten formulae of $N$ in $M$ are given respectively by $\widetilde{\nabla}_{X} Y=$ $\nabla_{X} Y+\sigma(X, Y)$ and $\tilde{\nabla}_{X} \xi=-A_{\xi} X+D_{X} \xi$, for $X, Y$ tangent to $N$ and $\xi$ normal to $N$. Moreover, the covariant derivative $\bar{\nabla} \sigma$ of $\sigma$ is defined by

$$
\left(\nabla_{X} \sigma\right)(X, Y)=D_{X} \sigma(Y, Z)-\sigma\left(\nabla_{X} Y, Z\right)-\sigma\left(Y, \nabla_{X} Z\right)
$$

We mention the following lemma for later use ([2]).

Lemma 3.1. Let $N$ be a submanifold of a Kaehler manifold. Then

(a) $\nabla P=0$ if and only if $A_{F X} Y=A_{F Y} X$, for all $X, Y$ tangent to $N$ and

(b) $\nabla F=0$ if and only if $A_{f \xi} X=-A_{\xi}(P X)$ for $\xi$ normal to $N$ and $X$ tangent to $N$.

REMARK 3.2. As usual, the covariant derivative of $F$ is defined by

$$
\left(\nabla_{X} F\right) Y=D_{X}(F Y)-F\left(\nabla_{X} Y\right), \quad \text { for } X, Y \text { in } T N .
$$

We recall that a submanifold $N$ of a Kaehler manifold $M$ is called generic if the maximal holomorphic subspace $\mathcal{H}_{x}=T_{x} N \cap J\left(T_{x} N\right)$ is of constant dimension. A submanifold is called minimal if $\operatorname{tr} \sigma=0$, and it is called austere if for each normal vector $\xi$ the set of eigenvalues of $A_{\xi}$ is invariant under multiplication by -1 ; this is equivalent to the condition that all the invariants of odd order of the Weingarten map at each normal to $N$ vanish. Of course an austere submanifold is a minimal submanifold.

LEMMA 3.3. Let $N$ be a submanifold of a Kaehler manifold $M$. Then we have:

(1) if $N$ is either totally real or holomorphic, then $F$ is parallel (that is, $\nabla F=0$ );

(2) if $N$ is generic and $F$ is parallel, then $F(T N)$ is a parallel subbundle of the normal bundle $T^{\perp} N$;

(3) if -1 is not an eigenvalue of $Q$ at any point of $N$ and $\nabla F=0$, then $\operatorname{Im} \sigma \subseteq F(T N)$;

(4) if 0 is not an eigenvalue of $Q$ at any point of $N$ and $\nabla F=0$, then $N$ is austere and hence $N$ is minimal.

Proof: Statement (1) is trivial. For statement (2), we assume $N$ is a generic submanifold of $M$ with $\nabla F=0$. Then the normal bundle $T^{\perp} N$ has the following orthogonal direct decomposition:

$$
T^{\perp} N=F(T N) \oplus \nu, \quad \nu_{x} \perp F\left(T_{x} N\right) .
$$

For any vector field $\xi$ in $\nu$ and $X, Y$ in $T N$, we have

$$
\begin{aligned}
\left\langle A_{J \xi} X, Y\right\rangle & =\langle\sigma\langle X, Y\rangle, J \xi\rangle=\left\langle\tilde{\nabla}_{X} Y, J \xi\right\rangle \\
& =-\left\langle P Y, A_{\xi} X\right\rangle+\left\langle F Y, D_{X} \xi\right\rangle
\end{aligned}
$$


from which we find

$$
\left\langle D_{X}(F Y) \xi\right\rangle=-\left\langle A_{\xi}(P Y)+A_{J \xi} Y, X\right\rangle .
$$

On the other hand, for any $\xi$ normal to $N$, if we denote by $t \xi$ and $f \xi$ the tangential and normal components of $J \xi$ respectively, then we have $[2, \mathrm{p} .264]$

$$
\left\langle\left(\nabla_{X} F\right) Y, \xi\right\rangle=-\left\langle A_{f \xi} Y+A_{\xi}(P Y), X\right\rangle .
$$

Since $f=J$ on $\nu,(3.3)$ and (3.4) imply $\left\langle D_{X}(F Y), \xi\right\rangle=0$ for any $\xi$ in $\nu$. This proves (2).

For (3), if $\nabla F=0$, Lemma 3.1 implies $\langle\sigma\langle X, Y\rangle, J \xi\rangle=-\langle\sigma(X, P Y), \xi\rangle$ for any $\xi$ in $\nu$. Thus, for any eigenvector $Y$ of $Q$ with eigenvalue $\lambda$ and any $\xi$ in $\nu$, we have $\langle\sigma(X, Y), \xi\rangle=-\lambda\langle\sigma(X, Y), \xi\rangle$. From this we obtain statement (3).

We now prove (4). Assume $\nabla F=0$. Then we have $f \sigma(X, Y)=\sigma(X, P Y)$ (see [2, p.264]). Let $X$ be any unit eigenvector of $Q$ with eigenvalue $\lambda \neq 0$. Then $X \star=$ $P X / \sqrt{-\lambda}$ is a unit vector perpendicular to $X$. Thus, $\sigma(X, X)=\sigma(P X, P X) / \lambda=$ $-\sigma(X \star, X \star)$, which implies (4)

PROPOSITION 3.4. Let $N$ be a proper slant submanifold of a Kaehler manifold. If $\nabla F=0$, then $N$ is austere and, in particular, minimal.

This result follows from statement (4) of Lemma 3.3.

Proposition 3.5. Let $N$ be an n-dimensional slant submanifold of $\mathrm{C}^{m}$. If $\nabla F=0$, then $N$ is contained in a complex linear subspace $C^{n}$ of $C^{m}$ as a minimal slant submanifold.

This result follows from statements (2), (3) and (4) of Lemma 3.3 and a reduction theorem of Erbarcher.

\section{EULER NUMBERS AND SLANT SURFACES}

In the following, we assume $N$ is a slant surface in the flat complex number space $C^{2}$ with slant angle $\theta$. For a unit vector field $e_{1}$ tangent to $N$, we put

$$
e_{2}=(\sec \theta) P e_{1}, \quad e_{3}=(\csc \theta) F e_{1}, \quad e_{4}=(\csc \theta) F e_{2} .
$$

Then $e_{1}=-(\sec \theta) P e_{2}, e_{1}, e_{2}, e_{3}$ and $e_{4}$ form an orthonormal frame such that $e_{1}$, $e_{2}$ are tangent to $N$ and $e_{3}, e_{4}$ normal to $N$. We put $h_{i j}^{r}=\left\langle\sigma\left(e_{i}, e_{j}\right), e_{r}\right\rangle, i, j=1,2$ and $r=3,4$ and denote by $G$ and $G^{D}$ the Gauss curvature and normal curvature of $N$ in $\mathrm{C}^{2}$ respectively. Then we have

$$
\begin{aligned}
G & =h_{11}^{3} h_{22}^{3}-\left(h_{12}^{3}\right)^{2}+h_{11}^{4} h_{22}^{4}-\left(h_{12}^{4}\right)^{2}, \\
G^{D} & =h_{11}^{3} h_{12}^{4}+h_{12}^{3} h_{22}^{4}-h_{12}^{3} h_{11}^{4}-h_{22}^{3} h_{12}^{4} .
\end{aligned}
$$


Since $N$ is compact, we have

$$
\int_{N} G d V=2 \pi \chi(N) \text { and } \int_{N} G^{D} d V=2 \pi \chi\left(T^{\perp} N\right) .
$$

LEMMA 4.1. If $N$ is a slant surface in $C^{2}$ then $G=G^{D}$ identically.

Proof: If $N$ is a slant surface in $C^{2}$, then $\nabla P=0$ by Proposition 2.4. Thus we have (Lemma 3.1 )

$$
A_{F, Y} Y=A_{F Y} X
$$

for $X, Y$ langent to $N$. Hence, by applying (4.1) and (4.5), we find

$$
h_{12}^{3}=h_{11}^{4}, \quad h_{22}^{3}=h_{12}^{4} \text {. }
$$

Therefore, by (4.2), (4.3) and (4.6), we obtain $G=G^{D}$.

We give the following consequence of Lemma 4.1.

Proposition 4.2. Let $N$ be a compact surface. Then we have:

(1) if the Euler number $\chi(N) \neq 0$, then $N$ admits no slant imbedding in $C^{2}$;

(2) if $\chi(N)=0$ then every slant immersion of $N$ in $\mathbb{C}^{2}$ is regularly homotopic to an imbedding;

(3) if $N$ has positive (or negative) Gauss curvature, then $N$ admits no slant inmersions into $\mathrm{C}^{2}$.

Proof: If $N$ admits a slant imbedding in $C^{2}$, then Lemma 4.1 implies $G=G^{D}$. Thus $\chi\left(T^{\perp} N\right)=\chi(N)$. Since $\chi\left(T^{\perp} N\right)=2 q(q$ the self-intersection number [7]) and $q=0$ for an imbedding, we obtain (1). Statement (2) follows from $\chi\left(T^{\perp} N\right)=\chi(N)=0$ and the fact that the self-intersection number is a regularly homotopic invariant. Now we prove (3). If $N$ is a compact slant surface with positive (or negative) Gauss curvature in $C^{2}$, then Lcmma 4.1 implies that $G^{D}$ is everywhere positive (or negative). Thus, by a result of Litle [9], $\chi\left(T^{\perp} N\right)=-2 \chi(N)$ (or $\chi\left(T^{\perp} N\right)=2 \chi(N)$ ) which is impossible unless $\chi(N)=0$. But this is a contradiction of the curvature condition.

\section{Minimal SLANT SURFACES}

In the following, $E^{4}$ denotes Euclidean 4-space with the standard metric. An almost complex structure $J$ on $E^{4}$ is said to be compatible if $\left(E^{4}, J\right)$ is a flat Kaehler manifold. We denote by $J_{0}$ the compatible almost complex structure on $E^{4}$ defined by

$$
J_{0}(a, b, c, d)=(-c,-d, a, b) .
$$

The flat Kaelder manifold $\left(E^{4}, J_{v}\right)$ is simply denoted by $C^{2}$. 
Lemma 5.1. If $N$ is a holomorphic surface in $C^{2}$, then, for any constant $\alpha$, $0<\alpha \leq \pi / 2, N$ is a slant surface in $\left(E^{4}, J_{\alpha}\right)$ with slant angle $\alpha$, where $J_{\alpha}$ is the compatible almost complex structure on $E^{4}$ defined by

$$
J_{\alpha}(a, b, c, d)=(\cos \alpha)(-c,-d, a, b)+(\sin \alpha)(-b, a, d,-c) .
$$

Proof: Follows from direct computation.

THEOREM 5.2. Let $N$ be an oriented slant surface in $C^{2}$. Then there is a compatible almost complex structure $\stackrel{\circ}{J}$ on $E^{4}$ such that $N$ is holomorphic in $\left(E^{4}, \stackrel{0}{J}\right)$ if and only if $N$ is minimal.

Proof: If $f: N \rightarrow C^{2}$ is a slant immersion, then $G=G^{D}$ (Lemma 4.1). Let $e_{1}, e_{2}$ be an oriented orthonormal frame on $N$. Then the Gauss map $\nu: N \rightarrow G(2,4)$ from $N$ into the real Grasmannian $G(2,4)$ is defined by $\nu(p)=\left(e_{1} \wedge e_{2}\right)(p)$ for $p \in N$. Denote by $S_{+}^{2}$ and $S_{-}^{2}$ the space of positive and negative sympletic 2-vectors in $\wedge^{2} E^{4}$ (see for instance, [5]). Then $G(2,4)$ is isometric to the Riemannian product $S_{+}^{2} \times S_{-}^{2}$. Denote by $\nu_{1}$ and $\nu_{2}$ the projections of $\nu$ into $S_{+}^{2}$ and $S_{-}^{2}$ respectively, so that $\nu=$ $\left(\nu_{1}, \nu_{2}\right): N \rightarrow S_{+}^{2} \times S_{-}^{2}$. Then $\nu_{1}$ is a holomorphic map (see for instance, [8]), if $f$ is minimal. Thus, if $N$ is minimal in $C^{2}$, the image $\nu_{1}(N)$ is either a point or an open subset of $S_{+}^{2}$. On the other hand, it is also known that the rank of $\nu_{1}$ is $\leq 1$ if and only if $G=G^{D}$ (Hoffman and Osserman; see [5]). Thus, if $f: N \rightarrow \mathbb{C}^{2}$ is minimal and slant, then $\nu_{1}$ is a constant map. Thus, by applying Proposition 2.2(i) of [5], $N$ is holomorphic with a certain compatible almost complex structure on $E^{4}$. The converse of this is trivial since every holomorphic surface in a Kaehler manifold is minimal.

REMARK 5.3. Theorem 5.2 generalises a result of $[4,5]$.

REMARK 5.4. T'heorem 5.2 implies that there exist many examples of slant surfaces in $C^{2}$.

The following result provides another characlerisation of minimal slant surfaces in $c^{2}$.

THEOREM 5.5. Let $N$ be a surface in $C^{2}$ which is neither holomorphic nor totally real. Then $N$ is a minimal slant surface if and only if $\nabla F=0$.

Proof: Since $N$ is neither holomorphic nor totally real in $\mathbb{C}^{2}$, both $P: T_{x} N \rightarrow$ $T_{x} N$ and $F: T_{x} N \rightarrow T_{x}^{\perp} N$ are surjective for each $x \in N$. Thus, if $\theta$ denotes the Wirtinger angle and $e_{1}$ a unit vector tangent to $N$, then $e_{1}, e_{2}=(\sec \theta) P e_{1}, e_{3}=$ $(\csc \theta) F e_{1}$ and $e_{4}=(\csc \theta) F e_{2}$ form an orthonormal frame. Since $J^{2}=-I$ these imply

$$
\begin{aligned}
& t e_{3}=-(\sin \theta) e_{1}, \quad t e_{4}=-(\sin \theta) e_{2}, \\
& f e_{3}=-(\cos \theta) e_{4}, \quad f e_{4}=(\cos \theta) e_{3} \text {. }
\end{aligned}
$$


If $\nabla F=0$, then, by Lemma 3.1 , we have $A_{f \xi} X=-A_{\xi}(P X)$ for any $\xi$ normal to $N$ and $X$ tangent to $N$. In particular, we have

$$
\begin{aligned}
A_{F e_{1}} e_{2} & =(\tan \theta) A_{e_{3}}\left(P e_{1}\right)=-(\tan \theta) A_{f e_{3}} e_{1} \\
& =(\sin \theta) A_{e_{4}} e_{1}=A_{F e_{2}} e_{1} .
\end{aligned}
$$

Therefore, by applying Lemma 3.1 again, we obtain $\nabla \cdot P=0$. Consequently, from Propositions 2.4 and $3.4, N$ is minimal and proper slant in $C^{2}$.

Conversely, if $N$ is minimal and proper slant, then (4.6) gives

$$
h_{11}^{3}=-h_{22}^{3}=-h_{12}^{4}, \quad h_{11}^{4}=-h_{22}^{4}=h_{12}^{3},
$$

from which by direct computation we may obtain $A_{f \xi} X=-A_{\xi}(P X)$ for any $\xi$ normal to $N$ and $X$ tangent to $N$. Thus, by applying Lemuma $3.1, F$ is also parallel.

REMARK 5.6. Theorem 5.5 still holds if $C^{2}$ is replaced by an arbitary Kaehler manifold of complex dimension 2 .

From Proposition 3.5 and Theorem 5.5 we have:

Corollary 5.7 . Let $N$ be a surface in $\mathbb{C}^{m}$. Then $\nabla F=0$ if and only if either $N$ is totally real or holomorphic in $C^{m}$ or $N$ is a minimal proper slant surface in a complex linear subspace $\mathrm{C}^{2}$ of $\mathrm{C}^{m}$.

\section{Totally REAL SURFACES}

THEOREM 6.1. Let $N$ be a proper slant surface in $\mathbb{C}^{2}$. Then there is a compatible almost complex structure $J_{1}$ on $E^{4}$ such that $N$ is totally real in $\left(E^{4}, J_{1}\right)$ if and only if $N$ is minimal.

Proof: Let $N$ be a proper slant surface in $C^{2}$. We may choose $e_{1}, e_{2}, e_{3}, e_{4}$ satisfying (4.1) and (5.3). Since $\widetilde{\nabla} J=0$, by comparing the normal components of $\tilde{\nabla}_{X}(J Y)$ and $J\left(\widetilde{\nabla}_{X} Y\right)$ and using the formulae of Gauss and Weingarten, we have

$$
D_{X}(F Y)-F\left(\nabla_{X} Y\right)=f \sigma(X, Y)-\sigma(X, P Y) .
$$

Thus, if we put $D e_{3}=\omega_{3}^{4} \otimes e_{4}$ and $D e_{4}=\omega_{4}^{3} \otimes e_{3},(4.1),(5.3)$ and (6.1) imply

$$
\begin{aligned}
& \omega_{3}^{4}\left(e_{1}\right)=\omega_{1}^{2}\left(e_{1}\right)=-(\cot \theta)\left(t r h^{3}\right), \\
& \omega_{3}^{4}\left(e_{2}\right)-\omega_{1}^{2}\left(e_{2}\right)=-(\cot \theta)\left(t r h^{4}\right) .
\end{aligned}
$$

Suppose that there is a compatible almost complex structure $J_{1}$ on $E^{4}$ such that $N$ is totally real in $\left(E^{4}, J_{1}\right)$. Then there is a function $\varphi$ such that

$$
\begin{aligned}
& e_{3}=(\cos \varphi) J_{1} e_{1}+(\sin \varphi) J_{1} e_{2}, \\
& \dot{e}_{4}=-(\sin \varphi) J_{1} e_{1}+(\cos \varphi) J_{1} e_{2} .
\end{aligned}
$$


Since $J_{1}$ is parallel, (5.4) implies

$$
\omega_{3}^{4}(X)=\left\langle\widetilde{\nabla}_{X} e_{3}, e_{4}\right\rangle=\omega_{1}^{2}(X)+X \varphi .
$$

Thus we get

$$
\omega_{3}^{4}=\omega_{1}^{2}+d \varphi
$$

Combining (6.2) and (6.4) we find

$$
\cot \theta\left\{\left(t r h^{3}\right) \omega^{1}+\left(t r h^{4}\right) \omega^{2}\right\}=-d \varphi,
$$

where $\omega^{1}, \omega^{2}$ denote the dual 1 -forms of $e_{1}, e_{2}$. Also, $(6.3)$ gives $J_{1} e_{1}=(\cos \varphi) e_{3}-$ $(\sin \varphi) e_{4}$ and $J_{1} e_{2}=(\sin \varphi) e_{3}+(\cos \varphi) e_{4}$. Thus, by $(6.3)$, we obtain

$$
h_{11}^{3}=\left(\cos ^{2} \varphi\right) h_{11}^{3}+\sin \varphi \cos \varphi\left(h_{11}^{4}-h_{12}^{3}\right)+\sin ^{2} \varphi h_{12}^{4} .
$$

Thus, by applying (4.6) we get

$$
\left(\sin ^{2} \varphi\right)\left(t r h^{3}\right)=0
$$

Sinilarly, we obtain

$$
\left(\sin ^{2} \varphi\right)\left(t r h^{4}\right)=0 .
$$

Let $U=\{x \in N \mid H(x) \neq 0\}$ where $H$ is the mean curvature vector. By (6.6) and (6.7), we get $\varphi \equiv 0(\bmod \pi)$ on $U$. Hence, by $(6.5), \cot \theta=0$ on $U$. This says that if $N$ is not minimal in $\mathbb{C}^{2}$, then $N$ is tolally real in $\mathbb{C}^{2}$ which contradicts the assumption. Thus, if $N$ is totally real in $\left(E^{4}, J_{1}\right)$ for some compatible $J_{1}$ then $N$ is minimal in $C^{2}$.

Conversely, if $N$ is minimal and proper slant in $C^{2}$, then, by Theorem $5.2, N$ is holomorphic in $E^{4}$ with respect to some compatible almost complex structure. Thus, by Lemma $5.1, N$ is totally real with respect to a suitable compatible complex structure.

Cumbining T'heorems 5.2 and 6.1 we have

THEOREM 6.2. Let $N$ be a totally real surface in $\mathbb{C}^{2}$. Then there is a compatible almost complex structure $J_{1}$ on $E^{4}$ such that $N$ is a proper slant surface in $\left(E^{4}, J_{1}\right)$ if and only if $N$ is minimal.

Proof: Let $N$ be a totally real surface in $C^{2}$. If $N$ is proper slant with respect to a compatible almost complex structure $J_{1}$ on $E^{4}$, then Theorem 6.1 implies that $N$ is minimal in $E^{4}$. Conversely, if $N$ is minimal, and totally real, then $N$ is proper slant with respect to a compatible almost complex structure by Theorem 5.2 and Lemma 5.1 . 


\section{Classification of slant surfaces With parallel MEAN CURVature vector}

Theorem 7.1. Let $N$ be a surface in $C^{2}$. Then $N$ is a slant surface with parallel mean curvature vector, that is $D H=0$, if and only if $N$ is one of the following surfaces:

(a) an open portion of the product surface of two plane circles, or

(b) an open portion of a circular cylinder contained in a hyperplane of $\mathrm{C}^{2}$, or

(c) a minimal slant surface in $\mathbb{C}^{2}$.

Moreover, in cases (a) and (b), $N$ is totally real in $\mathrm{C}^{2}$.

Proof: Let $N$ be a slant surface in $C^{2}$ with parallel mean curvature vector. Then the length of $H$ is constant. Assume $N$ is not minimal. 'Then one may choose $e_{1}$ such that $e_{3}=(\csc \theta) F e_{1}$ is in the direction of $I f$. (Such $e_{1}$ is chosen if we choose $e_{1}$ in the direction of $-t H$.) Since $D H=0, \omega_{3}^{4}=0$. Thus the normal curvature $G^{D}$ vanishes. Hence, by Lemma $4.1, N$ is flat. Now, let $V=\left\{x \in N \mid A_{\mathrm{e}_{4}} \neq 0\right.$ at $\left.\boldsymbol{x}\right\}$. If $V=\emptyset$, then $\operatorname{Im} \sigma \subseteq \operatorname{Span}\{H\}$. Thus, by $D H=0, N$ lies in a hyperplane $E^{3}$ of $C^{2}$. Since $N$ is flat with nonzero constant mean curvature, $N$ is an open portion of a circular cylinder. (See Proposition 3.2 of $[\mathbf{1}, p .118]$ ). If $V \neq \emptyset$ and $W$ is a connected component of $V$, then $e_{4}$ is a parallel, minimal, non-geodesic section on $W$. Since $W$ is flat, Proposition 5.4 of $[1, p .128]$ implies that $W$ is an open piece of the product surface of two plane circles. Since $\operatorname{det}\left(A_{e_{4}}\right)$ is a nonzero constant on $W$, by continuity, we have $V=N$. Thus, the whole surface is an open portion of the product surface.

Now, we claim that if $N$ is non-minimal, then $N$ is totally real in $C^{2}$. This can be seen as follows. If $N$ is not totally real, then, by $\omega_{3}^{4}=0$ and $t r h^{4}=0,(6.2)$ gives

$$
\omega_{1}^{2}\left(e_{2}\right)=0, \quad \omega_{1}^{2}\left(e_{1}\right)=(\cot \theta)\left(t r h^{3}\right)
$$

Because $N$ is flat, by the structure equation and (7.1) we obtain

$$
0=2 d \omega_{2}^{1}\left(e_{1}, e_{2}\right)=-\left(\omega_{2}^{1}\left(e_{1}\right)\right)^{2}
$$

Thus, by (7.1), we obtain $\cot \theta=0$ which implies $N$ is totally real.

The converse of this is easy to verify.

By applying Thoerem 7.1, we may obtain the following classification of slant surfaces with parallel second fundarnental form.

TheOREM 7.2. Let $N$ be a surface in $C^{2}$. Then $N$ is a slant surface in $C^{2}$ with parallel second fundamental form, that is $\bar{\nabla} \sigma=0$, if and only if $N$ is one of the following surfaces:

(a) an open portion of the product of two plane circles; 
(b) an open protion of a circular cylinder which is contained in a hyperplane of $\mathrm{C}^{2}$;

(c) an open portion of a slant plane in $\mathrm{C}^{2}$.

Moreover, in cases (a) and (b), $N$ is totally real in $C^{2}$.

Proof: If $N$ is a surface in $C^{2}$ with parallel second fundamental form, then $N$ has parallel mean surface vector. Thus, by Theorem 7.1 , it suffices to prove that slant planes are the only minimal slant surfaces with parallel second fundamental form. But this follows from the fact that every surface in $C^{2}$ with $\bar{\nabla} \sigma=0$ is locally symmetric and the only minimal surfaces in $E^{4}$ with constant Gauss curvature are the totally geodesic ones.

From Theorem 7.1 we obtain the following:

COROLlaRy 7.3. There exist no compact proper slant surfaces in $C^{2}$ with parallel mean curvature vector.

COROLlary 7.4. Let $N$ be a slant surface in $C^{2}$ with constant mean curvature. Then $N$ is spherical if and only if $N$ is an open portion of the product surface of two plane circles.

Proof: If $N$ is a spherical surface with constant mean curvature, then the mean curvature vector of $N$ in $C^{2}$ is parallel. Thus, by Theorem 7.1, $N$ is one of the surfaces mentioned in Theorem 7.1. Among them, surfaces of type (a) are the only spherical ones. The converse is clear.

\section{Some examples}

EXAMPLE 8.1. For any nonzero constants $a$ and $b$,

$$
x(u, v)=(a \cos u, b \cos v, a \sin u, b \sin v)
$$

gives a compact lotally real surface in $C^{2}$ with $\bar{\nabla} \sigma=0$.

EXAMPLE 8.2. For any $a>0$,

$$
x(u, v)=(a \cos u, v, a \sin u, 0)
$$

defines a non-compact totally real surface in $C^{2}$ with $\bar{\nabla} \sigma=0$.

EXAMPLE 8.3. For any $\alpha, 0<\alpha \leq \pi / 2$,

$$
x(u, v)=(u \cos \alpha, u \sin \alpha, v, 0)
$$

defines a slant plane with slant angle $\alpha$ in $C^{2}$. 
EXAMPLE 8.4. For any positive constant $k$,

$$
x(u, v)=\left(e^{k u} \cos u \cos v, e^{k u} \sin u \cos v, e^{k u} \cos u \sin v, e^{k u} \sin u \sin v\right)
$$

defines a complete, non-rninimal, psuedo-umbilical proper slant surface in $C^{2}$ with slant angle $\cos ^{-1}\left(k / \sqrt{1+k^{2}}\right)$ and with mean curvature $e^{-k u} / \sqrt{1+k^{2}}$.

EXAMPLE 8.5. For any positive number $k$,

$$
x(u, v)=(u, k \cos v, v, k \sin v)
$$

defines a complete, flat, non-minimal and non-psuedo-umbilical, proper slant surface with slant angle $\cos ^{-1}\left(1 / \sqrt{1+k^{2}}\right)$ and constant mean curvature $k / 2\left(1+k^{2}\right)$ and with non-parallel mean curvature vector.

EXAMPLE 8.6. Let $k$ be any positive number and $(g(s), h(s))$ be a unit speed plane curve. Then

$$
x(u, s)=(-k s \sin u, g(s), k s \cos u, h(s))
$$

defines a non-minimal, flat, proper slant surface with slant angle $k / \sqrt{1+k^{2}}$.

Finally, we give some examples of Kaehlerian slant submanifolds in $\mathbb{C}^{4}$.

EXAMPLE 8.7. For any $k>0$,

$$
x(u, v, w, z)=(u, v, k \sin w, k \sin z, k w, k z, k \cos w, k \cos z)
$$

defines a Kaehlerian slant submanifold in $C^{4}$ with slant angle $\cos ^{-1} k$.

Added in proof. In a forthcoming article of the author and $\mathrm{Y}$. Tazawa it is proved that every compact slant submanifold of $\mathbb{C}^{m}$ is totally real.

\section{REFERENCES}

[1] B.Y. Chen, Geometry of Submanifolds (Dekker, New York, 1973).

[2] B.Y. Chen, 'Differential geometry of real submanifolds in a Kähler manifold', Monatsh. Math. 81 (1981), 257-274.

[3] B.Y. Chen and K. Ogiue, 'On totally real submanifolds', Trans. Amer. Math. Soc. 193 (1974), 257-266.

[4] B.Y. Chen and J.M. Morvan, 'Propriété riemanniennes des surfaces lagrangiennes', C.R. Acad. Sci. Paris. Ser I 301 (1985), 209-212.

[5] B.Y. Chen and J.M. Morvan, 'Geometrie des surfaces lagrangiennes de $C^{2}$, J. Math. Pures Appl. 66 (1987), 321-335.

[6] L. Gheysens, P. Verheyen and L. Verstraelen, 'Characterization and examples of Chen subinanifolds', J. Geom. 20 (1983), 47-62. 
[7] R.K. Lashof and S. Smale, 'On the immersion of manifolds in Euclidean spaces', Ann. of Math. 68 (1958), 562-583.

[8] H.B. Lawson, Lectures on Minimal Submanifolds (Publish or Perish, Berkeley, 1980).

[9] J.A. Little, 'On singularities of surfaces in $E^{4}$, Bull. Amer. Math. Soc. 75 (1969), 368-369.

Department of Mathematics

Michigan State University

East Lansing MI 48824

United States of America. 